\title{
Magnetic stimulation in the microscale: the development of a $6 \times 6$ array of micro-coils for stimulation of excitable cells in vitro
}

To cite this article before publication: Maria Eleni Rizou et al 2017 Biomed. Phys. Eng. Express in press https://doi.org/10.1088/20571976/aaa0dd

\author{
Manuscript version: Accepted Manuscript \\ Accepted Manuscript is "the version of the article accepted for publication including all changes made as a result of the peer review process, \\ and which may also include the addition to the article by IOP Publishing of a header, an article ID, a cover sheet and/or an 'Accepted \\ Manuscript' watermark, but excluding any other editing, typesetting or other changes made by IOP Publishing and/or its licensors" \\ This Accepted Manuscript is () 2017 IOP Publishing Ltd
}

During the embargo period (the 12 month period from the publication of the Version of Record of this article), the Accepted Manuscript is fully protected by copyright and cannot be reused or reposted elsewhere.

As the Version of Record of this article is going to be / has been published on a subscription basis, this Accepted Manuscript is available for reuse under a CC BY-NC-ND 3.0 licence after the 12 month embargo period.

After the embargo period, everyone is permitted to use copy and redistribute this article for non-commercial purposes only, provided that they adhere to all the terms of the licence https://creativecommons.org/licences/by-nc-nd/3.0

Although reasonable endeavours have been taken to obtain all necessary permissions from third parties to include their copyrighted content within this article, their full citation and copyright line may not be present in this Accepted Manuscript version. Before using any content from this article, please refer to the Version of Record on IOPscience once published for full citation and copyright details, as permissions will likely be required. All third party content is fully copyright protected, unless specifically stated otherwise in the figure caption in the Version of Record.

View the article online for updates and enhancements. 


\title{
1. Introduction
}

Precise control over the activity of a large number of excitable cells, such as neural cells, has been a long-term goal of the scientific community. Until now, different approaches have been suggested for stimulation of neural cell and tissue, such as electric, optical, thermal and magnetic stimulation. Electric stimulation is probably the most known method[1,2] where a potential difference is applied between two, or more, electrodes to excite cell activity. Electric stimulation has been performed in application of in vitro cell culturing, while in vivo approaches vary in the extend of invasiveness. There are many commercially available platforms for electric stimulation, which face the problems of over time electrode degradation and corrosion, biofouling and phenomena occurring in the interface between electrode-electrolyte which limit their capabilities. To overcome corrosion problems, platforms for electrical stimulation with high-k dielectric insulation have been proposed [3]. In optical stimulation, on the other hand the source of activation is light. Optogenetics is a promising and relatively new way of neural cell stimulation[4], which has attracted significant attention over the past years. However, since cells are not responding to light signals, they need to undergo a photosensitization procedure prior to their exposure to stimuli. This procedure usually involves the copy and transfer of the appropriate genes to the cells, adding complexity to the method, while at the same time, raising issues about the possible future application to humans[5]. Thermal stimulation has also been proposed as a successful stimulation method, where a localized gradient of temperature is created near the cell area. Different ways to create the localized gradient of temperature have been proposed[6,7].

Transcranial magnetic stimulation (TMS) is used to elicit neuronal activity in the brain, non-invasively and has been developed over the past decades [8-11]. TMS is used for the clinical treatment of a number of different diseases, from Parkinson disease, Alzheimer and Schizophrenia to depression. In this method, a magnetic coil

is used externally to the scalp in order to generate a magnetic field, which easily passes through the skull and

\begin{abstract}
In this study we present the development of a prototype device, designed for micro-magnetic stimulation

of excitable cells in vitro. Each platform consists of a $6 \mathrm{x} 6$ two-dimensional array of micro-coils, in an
attempt to achieve highly localised magnetic flux patterns. The coils are fabricated with standard micro-
fabrication techniques, including steps of photolithography, dry etching and electroplating. The further
interfacing of the micro-magnetic chip into a biocompatible platform is also described. The samples are
characterised electrically, while a finite element method simulation study is performed and reveals a
$141 \mathrm{mV}$-strong electric potential induced in the vicinity of a micro-coil. Since applications in neuronal
cells is our primary focus, modelling with NEURON software is used for demonstrating the capability
of the platform to activate adjacent cells. Finally, an experimental yalidation of the proof of concept is
performed with the measurement of induced current into a custom-made phantom gel that shows similar
electric properties with brain tissue.

of excitable cells in vitro. Each platform consists of a $6 \mathrm{x} 6$ two-dimensional array of micro-coils, in an
attempt to achieve highly localised magnetic flux patterns. The coils are fabricated with standard micro-
fabrication techniques, including steps of photolithography, dry etching and electroplating. The further
interfacing of the micro-magnetic chip into a biocompatible platform is also described. The samples are
characterised electrically, while a finite element method simulation study is performed and reveals a
$141 \mathrm{mV}$-strong electric potential induced in the vicinity of a micro-coil. Since applications in neuronal
cells is our primary focus, modelling with NEURON software is used for demonstrating the capability
of the platform to activate adjacent cells. Finally, an experimental yalidation of the proof of concept is
performed with the measurement of induced current into a custom-made phantom gel that shows similar
electric properties with brain tissue.

of excitable cells in vitro. Each platform consists of a $6 \mathrm{x} 6$ two-dimensional array of micro-coils, in an
attempt to achieve highly localised magnetic flux patterns. The coils are fabricated with standard micro-
fabrication techniques, including steps of photolithography, dry etching and electroplating. The further
interfacing of the micro-magnetic chip into a biocompatible platform is also described. The samples are
characterised electrically, while a finite element method simulation study is performed and reveals a
$141 \mathrm{mV}$-strong electric potential induced in the vicinity of a micro-coil. Since applications in neuronal
cells is our primary focus, modelling with NEURON software is used for demonstrating the capability
of the platform to activate adjacent cells. Finally, an experimental yalidation of the proof of concept is
performed with the measurement of induced current into a custom-made phantom gel that shows similar
electric properties with brain tissue.

of excitable cells in vitro. Each platform consists of a $6 \mathrm{x} 6$ two-dimensional array of micro-coils, in an
attempt to achieve highly localised magnetic flux patterns. The coils are fabricated with standard micro-
fabrication techniques, including steps of photolithography, dry etching and electroplating. The further
interfacing of the micro-magnetic chip into a biocompatible platform is also described. The samples are
characterised electrically, while a finite element method simulation study is performed and reveals a
$141 \mathrm{mV}$-strong electric potential induced in the vicinity of a micro-coil. Since applications in neuronal
cells is our primary focus, modelling with NEURON software is used for demonstrating the capability
of the platform to activate adjacent cells. Finally, an experimental yalidation of the proof of concept is
performed with the measurement of induced current into a custom-made phantom gel that shows similar
electric properties with brain tissue.

of excitable cells in vitro. Each platform consists of a $6 \mathrm{x} 6$ two-dimensional array of micro-coils, in an
attempt to achieve highly localised magnetic flux patterns. The coils are fabricated with standard micro-
fabrication techniques, including steps of photolithography, dry etching and electroplating. The further
interfacing of the micro-magnetic chip into a biocompatible platform is also described. The samples are
characterised electrically, while a finite element method simulation study is performed and reveals a
$141 \mathrm{mV}$-strong electric potential induced in the vicinity of a micro-coil. Since applications in neuronal
cells is our primary focus, modelling with NEURON software is used for demonstrating the capability
of the platform to activate adjacent cells. Finally, an experimental yalidation of the proof of concept is
performed with the measurement of induced current into a custom-made phantom gel that shows similar
electric properties with brain tissue.

of excitable cells in vitro. Each platform consists of a $6 \mathrm{x} 6$ two-dimensional array of micro-coils, in an
attempt to achieve highly localised magnetic flux patterns. The coils are fabricated with standard micro-
fabrication techniques, including steps of photolithography, dry etching and electroplating. The further
interfacing of the micro-magnetic chip into a biocompatible platform is also described. The samples are
characterised electrically, while a finite element method simulation study is performed and reveals a
$141 \mathrm{mV}$-strong electric potential induced in the vicinity of a micro-coil. Since applications in neuronal
cells is our primary focus, modelling with NEURON software is used for demonstrating the capability
of the platform to activate adjacent cells. Finally, an experimental yalidation of the proof of concept is
performed with the measurement of induced current into a custom-made phantom gel that shows similar
electric properties with brain tissue.

of excitable cells in vitro. Each platform consists of a $6 \mathrm{x} 6$ two-dimensional array of micro-coils, in an
attempt to achieve highly localised magnetic flux patterns. The coils are fabricated with standard micro-
fabrication techniques, including steps of photolithography, dry etching and electroplating. The further
interfacing of the micro-magnetic chip into a biocompatible platform is also described. The samples are
characterised electrically, while a finite element method simulation study is performed and reveals a
$141 \mathrm{mV}$-strong electric potential induced in the vicinity of a micro-coil. Since applications in neuronal
cells is our primary focus, modelling with NEURON software is used for demonstrating the capability
of the platform to activate adjacent cells. Finally, an experimental yalidation of the proof of concept is
performed with the measurement of induced current into a custom-made phantom gel that shows similar
electric properties with brain tissue.

of excitable cells in vitro. Each platform consists of a $6 \mathrm{x} 6$ two-dimensional array of micro-coils, in an
attempt to achieve highly localised magnetic flux patterns. The coils are fabricated with standard micro-
fabrication techniques, including steps of photolithography, dry etching and electroplating. The further
interfacing of the micro-magnetic chip into a biocompatible platform is also described. The samples are
characterised electrically, while a finite element method simulation study is performed and reveals a
$141 \mathrm{mV}$-strong electric potential induced in the vicinity of a micro-coil. Since applications in neuronal
cells is our primary focus, modelling with NEURON software is used for demonstrating the capability
of the platform to activate adjacent cells. Finally, an experimental yalidation of the proof of concept is
performed with the measurement of induced current into a custom-made phantom gel that shows similar
electric properties with brain tissue.

of excitable cells in vitro. Each platform consists of a $6 \mathrm{x} 6$ two-dimensional array of micro-coils, in an
attempt to achieve highly localised magnetic flux patterns. The coils are fabricated with standard micro-
fabrication techniques, including steps of photolithography, dry etching and electroplating. The further
interfacing of the micro-magnetic chip into a biocompatible platform is also described. The samples are
characterised electrically, while a finite element method simulation study is performed and reveals a
$141 \mathrm{mV}$-strong electric potential induced in the vicinity of a micro-coil. Since applications in neuronal
cells is our primary focus, modelling with NEURON software is used for demonstrating the capability
of the platform to activate adjacent cells. Finally, an experimental yalidation of the proof of concept is
performed with the measurement of induced current into a custom-made phantom gel that shows similar
electric properties with brain tissue.

of excitable cells in vitro. Each platform consists of a $6 \times 6$ two-dimensional array of micro-coils, in an
attempt to achieve highly localised magnetic flux patterns. The coils are fabricated with standard micro-
fabrication techniques, including steps of photolithography, dry etching and electroplating. The further
interfacing of the micro-magnetic chip into a biocompatible platform is also described. The samples are
characterised electrically, while a finite element method simulation study is performed and reveals a
$141 \mathrm{mV}$-strong electric potential induced in the vicinity of a micro-coil. Since applications in neuronal
cells is our primary focus, modelling with NEURON software is used for demonstrating the capability
of the platform to activate adjacent cells. Finally, an experimental yalidation of the proof of concept is
performed with the measurement of induced current into custom-made phantom gel that shows similar
electric properties with brain tissue.
\end{abstract}

1 Magnetic stimulation in the microscale: the development of a $6 \times 6$ array of micro2 coils for stimulation of excitable cells in vitro

\section{$3 \quad$ M E Rizou and T Prodromakis}

Nanoelectronics and Nanotechnology Research Group, Department of Electronics and Computer Science, e-mail: m.rizou@soton.ac.uk, t.prodromakis@soton.ac.uk

Abstract 
induces electric currents that stimulate the targeted brain region. Over the years, modelling and circuit design considerations for systems of micro-magnetic stimulation have been proposed [12-16]. Recent studies have demonstrated that millimetre or sub-millimetre electromagnets, can activate neuronal tissue or cells both in vitro and in vivo[17-20], bringing in the foreground the possibility that micro-magnetic stimulation coils, small enough to be implanted within the brain, may prove to be an effective alternative to existing stimulation devices.

The focus of this study is the scaling of TMS to the microscale, with the use of an array of electrically addressable micro-coils, designed to create spatiotemporal magnetic flux profiles on demand. The main challenge of this novel non-invasive neuro-stimulation tool is that micro-coils need to hold certain current capacity, so as to induce an electric field strong enough to stimulate cells, as cells need to reach their threshold potential in order to fire. Specifically, we present all the steps towards the design and construction of a micromagnetic platform for magnetic stimulation in vitro. The realization of a planar $6 \times 6$ array of micro-coil is important so as to cover a wider area of neural cells in a cell culture. The micro-coils in the arrays are creating magnetic flux density profiles with a spatial resolution equivalent to the micro-coil size, creating in such a way a non-invasive equivalent of a micro-electrode array. Each micro-coil is addressed individually and all the micro-coils of an array create equivalent magnetic flux profiles. Besides the fabrication development, different studies were used to characterize the behavior of the micro-arrays. Simulations with a finite element method (FEM) software and modeling with NEURON software, are used as a validation of the capability of the platform to excite neuronal activity. Additionally, the testing of the micro-coil with a phantom gel, which mimics the electric properties of brain tissue, is presented, as a first step towards the bio-experiment. The electric currents induced in the phantom gel during the activation of the micro-coils are measured and compared with other successful mechanisms of extracellular electric stimulation.

\section{Methods}

\subsection{Micro-fabrication procedure}

The procedure that was followed in order to fabricate the micro-inductors on a chip is illustrated in figure 1, for the case of a single coil and consists of two photolithographic steps. Firstly, a thin layer of Au is deposited, playing the role of a ground plane for the micro-coils of the array. The ground plane is made of Au and is deposited by an electron beam evaporator in the thickness of 50nm. The use of a ground plane simplifies the configuration in the two dimensional array as every coil will need a single pad connection instead of. It also enables the galvanic connection of all the metallic structures in a wafer piece to the same electric potential, during the electroplating step. A $5 \mathrm{~nm}$ thick $\mathrm{Cr}$ layer was added for adhesion purposes. On top of the ground plane an insulating layer of SiN is deposited with a thickness of $100 \mathrm{~nm}$ by reactive sputtering. The first optical lithography in combination with inductively coupled plasma (ICP) etching of SiN creates the central vias of the micro-coils, which enable the electrical connection of every coil to the Au ground plane. A second optical lithography and the lift-off of a 100nm Au seed layer defines the metallic tracks of the coils on top of the dielectric. Electroplating is then used to further increase the micro-coils' thickness in the micro scale. The results presented below concern micro-coils with a thickness of $1.7 \mu \mathrm{m}$. After this stage the chip is wirebonded to the packaging, an electrical insulating epoxy of high viscosity is used to protect the fragile wirebonds and to place a cell culturing well on top of the packaging. The high viscosity is important, so as to avoid epoxy to spread on the effective area of the chip and cover the micro-magnetic array. The cell culturing well is needed so as to define the cell medium during the bio-experiment. The whole chip (plus the effective area) is finally covered with a $800 \mathrm{~nm}$ thick layer of Parylene-C so as to create a biocompatible surface. The film of Parylene-C assures not only the biocompatibility of the surface, but also the electrical insulation of all the conducting parts left exposed (patterned micro-coils). At the same time, the thickness of the Parylene-C 
layer defines the distance between the micro-coils and the cell medium. The magnetic field is stronger in the vicinity of the micro-coils and this is why the deposited Parylene-C film is chosen to be relatively thin.

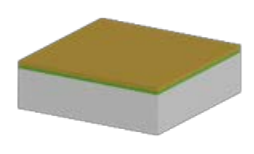

1. $\mathrm{Cr}, \mathrm{Au}$ deposition

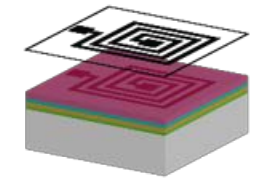

5. UV photolithography

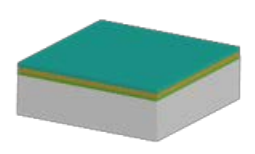

2. SiN deposition

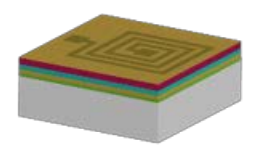

6. Au deposition

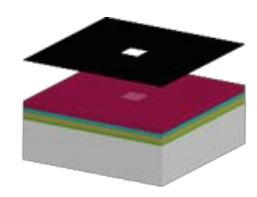

3. UV photolithography

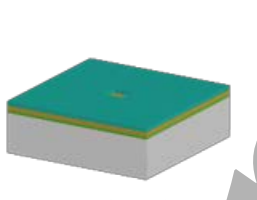

4. Opening vias

$\mathrm{Cr}$
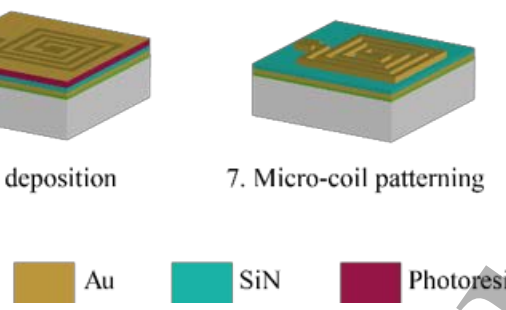

$\mathrm{SiN}$

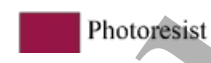

Figure 1. Schematic representation of the developed process flowchart for prototyping our micro-coil platform.

\subsection{FEM calculations}

A series of calculations were performed using COMSOL Multiphysics software. The design of the micro-coil geometry, used for the fabrication of the photolithography mask, was imported to COMSOL 4.4 using the AC/DC module. The 3-D model consists of a single micro-coil geometry on top of a Si wafer and positioned inside a uniform volume of air, representing the surrounding during the electrical testing. The biasing signal of the micro-coils was set $200 \mathrm{mV}$ in magnitude was solved in the frequency domain.

\subsection{Electric measurements}

An impedance sweep of our device was performed on a Kéithley semiconductor parameter analyser. The testing biasing signal was $30 \mathrm{mV}$ and three set of measurements were taken. The resistance and reactance of the devices are calculated by measurements of the impedance magnitude and phase angle, while the inductance is extracted through the obtained values of reactance.

\subsection{Modelling with NEURON software}

NEURON simulation environment[21], is used to create a model for simulating the effects of magnetic stimulation in a neural cell. As described in study[14], assuming a neural axon lying parallel to the plane of the micro-coil, the electric field which is induced in the vicinity of the coil could be expressed as a product of a spatial and a temporal component. The spatial component of the induced electric field in the $\mathrm{x}$ direction can be calculated externally and then inserted to NEURON code. As our platform should work over a wide range of biological preparations and can be used versatilely with different cell cultures or tissue slices, a variety of cell morphologies is expected. Throughout this study, we employed the model presented in[14] and modified this to account for an axon with a length of $70 \mu \mathrm{m}$ and diameter of $10 \mu \mathrm{m}$, comparable to the dimensions of the microcoil. The axon is centred above the coil and is assigned active Hodgkin-Huxley channels. The exported values of Ex(x) from COMSOL, for constant values of y (direction of axon in the xy plane) and $z$ (vertical distance of axon from the plane of micro-coil), are then inserted to NEURON to calculate the spatial derivative of Ex in the direction of $x$ and include it in the calculations of the induced electric field term in the cable equation. The cell $60^{123}$ is divided into discrete segments, on each of which an induced electric current is calculated, representing the magnetic stimulation effect on the segment. The temporal part of the solution, is calculated as described in the 


\section{Results}

Table 1. Phantom gel materials and composition.

methods of study[14] in every time step. The R, L, C values of the stimulator circuit are also modified to the measured values of our equivalent circuit, obtained from the electric characterization of the system presented in section 3.3. More specifically, the values of the RLC equivalent circuit used are: $\mathrm{R}=22 \mathrm{Ohms}, \mathrm{L}=1.69 \mu \mathrm{H}$ and $\mathrm{C}=14 \mathrm{nF}$, considering the self-resonant frequency at $10 \mathrm{MHz}$. Finally, the input stimulus has amplitude up to $200 \mathrm{mV}$ and stimulus durations between of $2 \mu \mathrm{s}-2 \mathrm{~ms}$.

\subsection{Fabrication of the phantom gel}

A phantom gel is a solution was fabricated in the lab so as to mimic the electric properties (electric permittivity and conductivity) of brain tissue. The phantom solution consistency is based on a previous study[22], however certain changes in the ingredients were needed so as to have a better fitting between the electrical properties of the gel and those of measured brain tissue for a lower frequency range. Additionally, an increase in the amount of the gelling agent hydroxy-ethyl cellulose (HEC) is introduced to achieve a semi-solid consistency, which is needed in our experiments. The phantom gel materials, used in the experiments below are presented in Table 1.

\begin{tabular}{lc}
\hline & \% by weight \\
\hline Sugar & 54.4 \\
Water & 39.2 \\
HEC & 3.9 \\
Salt $(\mathrm{NaCl})$ & 2.4 \\
Bacteriacide & 0.1 \\
\hline
\end{tabular}

\subsection{The fabricated prototype and the electric setup of the stimulator}

A scanning electron microscope (SEM) image of a single fabricated device and an example of a 6x6 array of micro-coils is presented in figure 2 (a) and (b) respectively. The micro-coil presented here, has a deterioration threshold regarding the current capacity it could hold and this is calculated at $20 \mathrm{~mA}$, as described on study [23]. Figure 2(c) shows the finalised prototype of our platform where the cell culture well is placed. The micro-array, located at the centre, is left clear of epoxy. A printed circuit board designed in house enables the individual activation of each coil on the packaged device.
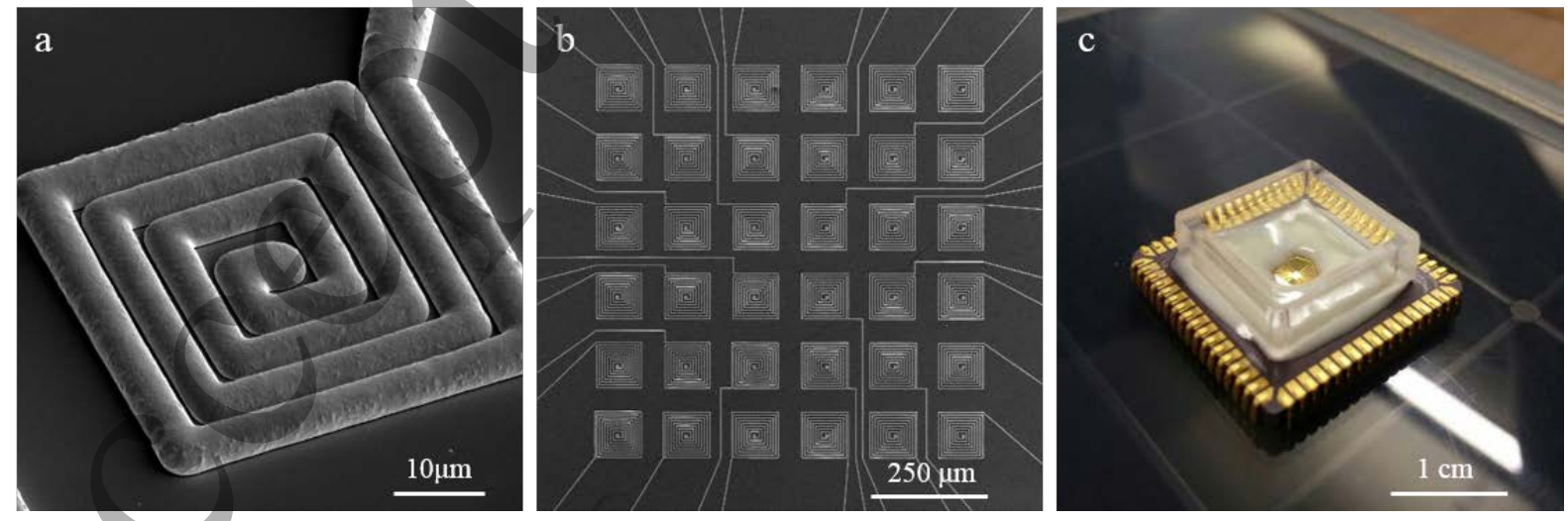

Figure 2. Illustrations of the fabricated platforms (a) SEM microphotograph of a single planar micro-coil and 
The activation setup, consists of a function generator, used to deliver a pulsed input signal to each micro-coil.

\subsection{FEM simulations}

Table 2. Geometric characteristics of our micro-coils.

\begin{tabular}{lcc}
\hline & Value & Unit \\
\hline Wire thickness & 1.7 & $\mu \mathrm{m}$ \\
Wire width & 2 & $\mu \mathrm{m}$ \\
Spacing & 2 & $\mu \mathrm{m}$ \\
Number of turns & 5 & \\
Surface area of coil & $50 \mathrm{x} 50$ & $\mu \mathrm{m}^{2}$ \\
\hline
\end{tabular}

The planar distribution of the magnetic flux density produced by the micro-coil is presented in figure 3(a). The distance is chosen based on the distance where the cell medium will be realistically placed, above the Parylene$\mathrm{C}$ encapsulation layer. As expected, the magnetic flux density maximum is above the centre of the micro-coil and reaches a value of $9.17 \mathrm{mT}$ at $800 \mathrm{~nm}$ above the coil. Since the small size of the inductors is a limiting factor for the characterization of their magnetic performance, the computational method is a useful tool to identify the magnetic behaviour of the coils.

a

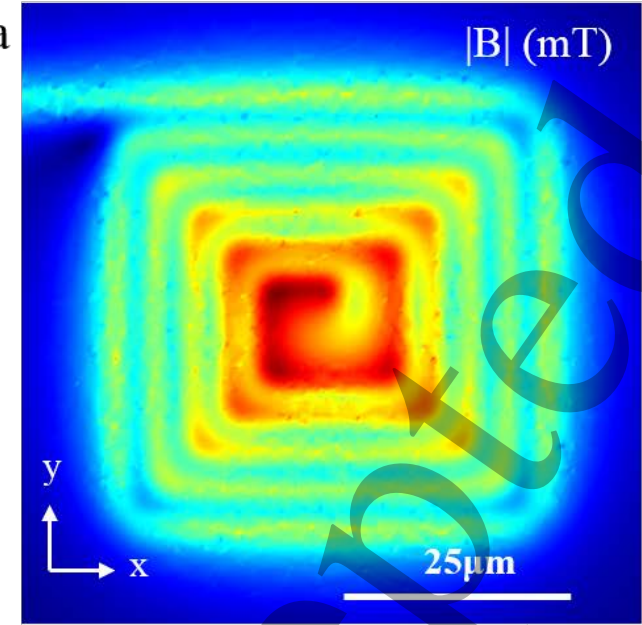

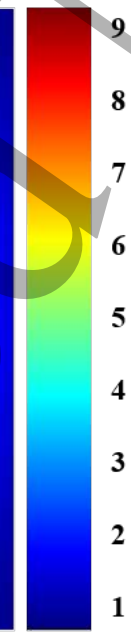
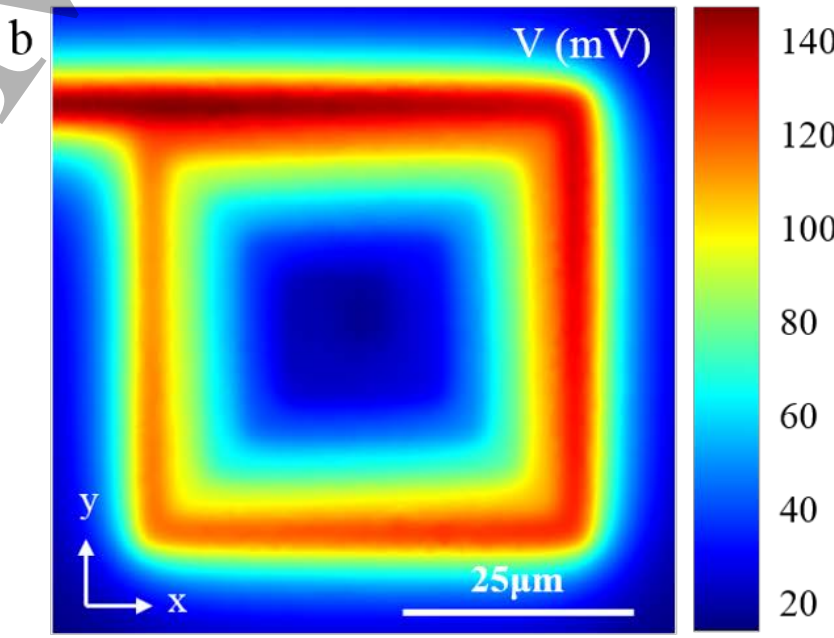

Figure 3(a) Planar distribution of the magnetic flux density in a distance $\mathrm{z}=800 \mathrm{~nm}$ above the micro-coil. (b) Planar distribution of induced electric voltage above the micro-coil at $\mathrm{z}=3 \mu \mathrm{m}$.

The spatial distribution of the electric voltage induced by the micro-coil is also presented in figure 3(b), at the xy plane at a distance of $3 \mu \mathrm{m}$ above the micro-coil. The induced electric potential or the induced electric field, calculated by Faraday's law of induction given in equation (1), is an important figure of merit for this device.

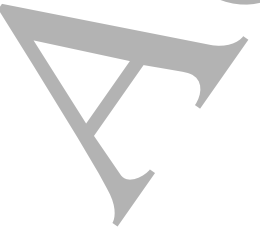



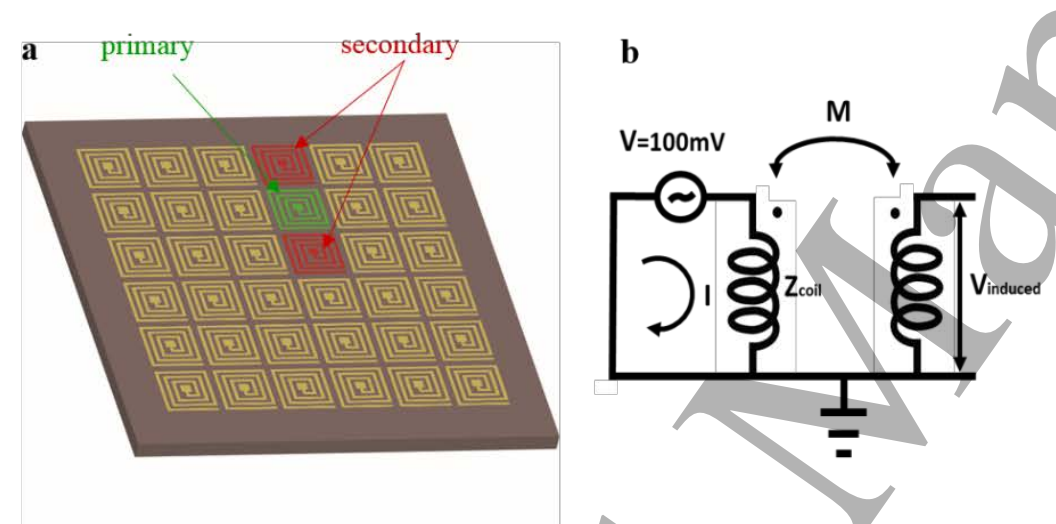

\subsection{Inductive coupling between micro-coils}

The activation of one micro-coil in the array, is expected to induce a voltage in adjacent micro-coils within the array. In order to quantify the effect of crosstalk we perform an experiment where a micro-coil (primary) is biased with a known sinusoidal signal and the voltage is measured in real time at first neighbouring micro-coils (secondary).

$$
\nabla \times \mathbf{E}=-\frac{\partial \mathbf{B}}{\partial \mathrm{t}}
$$

$\mathbf{E}$ and $\mathbf{B}$ are the vectors of the electric and magnetic field respectively.

The spatial derivative of the electric field in $\mathrm{x}$ and $\mathrm{y}$ directions is calculated and has a maximum of $\mathrm{dE}_{\mathrm{x}} / \mathrm{dx}=\sim 10^{9} \mathrm{~V} / \mathrm{m}^{2}$ at a distance $\mathrm{z}=5 \mu \mathrm{m}$ away from the micro-coil, well above the value of $11 \mathrm{kV} / \mathrm{m}^{2}$ which has been reported in $[20,24]$ as the threshold of stimulation for peripheral axons. This result corresponds to a current input of 9mA. The reason behind the significantly higher value from the threshold arises from the possibility of placing the cells in great proximity to the coil. In terms of fabrication, the proximity is mainly determined by the thickness of the Parylene-C insulating layer which covers the micro-coil. As a result, even with a significantly lower current amplitude from 9mA, we could still be above the activation threshold.

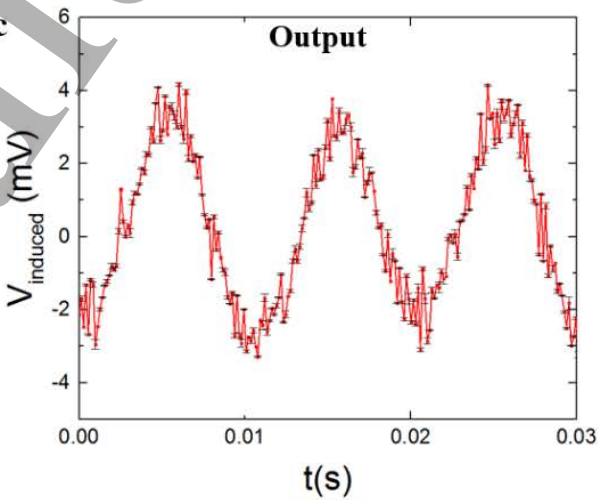

Figure 4. The inductive measurement methodology and results. (a) Primary activated micro-coil in the array, shown in green and secondary recording micro-coils in red. (b) Schematic of inductive measurement methodology. (c) The recorded output for a sinusoidal input, arising as an average of two simultaneous recordings at the secondary coils.

Figure 4a shows an example of the primary activated coil in the array, in green and the secondary ones, in red. The schematic of figure $4 \mathrm{~b}$, explains the methodology of an inductive measurement. The signal in the secondary coils is recorded for a sinusoidal input at an arbitrary frequency of $100 \mathrm{~Hz}$ and peak to peak value of $100 \mathrm{mV}$. The measurements were performed with a four probe system and a semiconductor parameter analyser. The recorded waveform from the secondary coils is presented in figure 4c. The recorded waveform, result from the average between two simultaneous recordings on two adjacent coils of similar separation distance. When a sinusoidal current (I) flows through the primary coil, the voltage in the secondary (V) in the frequency domain is described by equation (2).

$$
\mathrm{V}=\mathrm{i} \omega \mathrm{MI}
$$

Where $\mathrm{M}$ is the mutual inductance and $\omega$ the angular frequency of the signal. The result indicates that for an input signal with a peak to peak value of $100 \mathrm{mV}$, the induced voltage in the secondary coil decreases 60 
further the input signal frequency to $\mathrm{kHz}-\mathrm{MHz}$ range, however equation 2 clearly shows that the increase of frequency results in lower values of mutual inductance.

\subsection{Electric characterization of fabricated chip}

The fabricated chip was electrically characterised so as to identify the RLC equivalent circuit which describes its behaviour. A frequency sweep was performed, over a frequency range from $500 \mathrm{kHz}-10 \mathrm{MHz}$, based on which the inductance and quality factor of the system are extracted. In figure 5(a), the impedance magnitude and the phase angle of the complex impedance vector are presented. Figure 5(b), shows the extracted values of inductance and quality factor $\mathrm{Q}$, during the electrical test.

Observing the behaviour of $Z$ amplitude and phase in figure 5 (a), we notice that the self resonant frequency of the system, should be at around $10 \mathrm{MHz}$, as $\mathrm{Z}$ magnitude reaches a peak and phase starts to decrease after a plateau. Above the self resonant frequency, the capacitance behaviour of the system would be dominant.
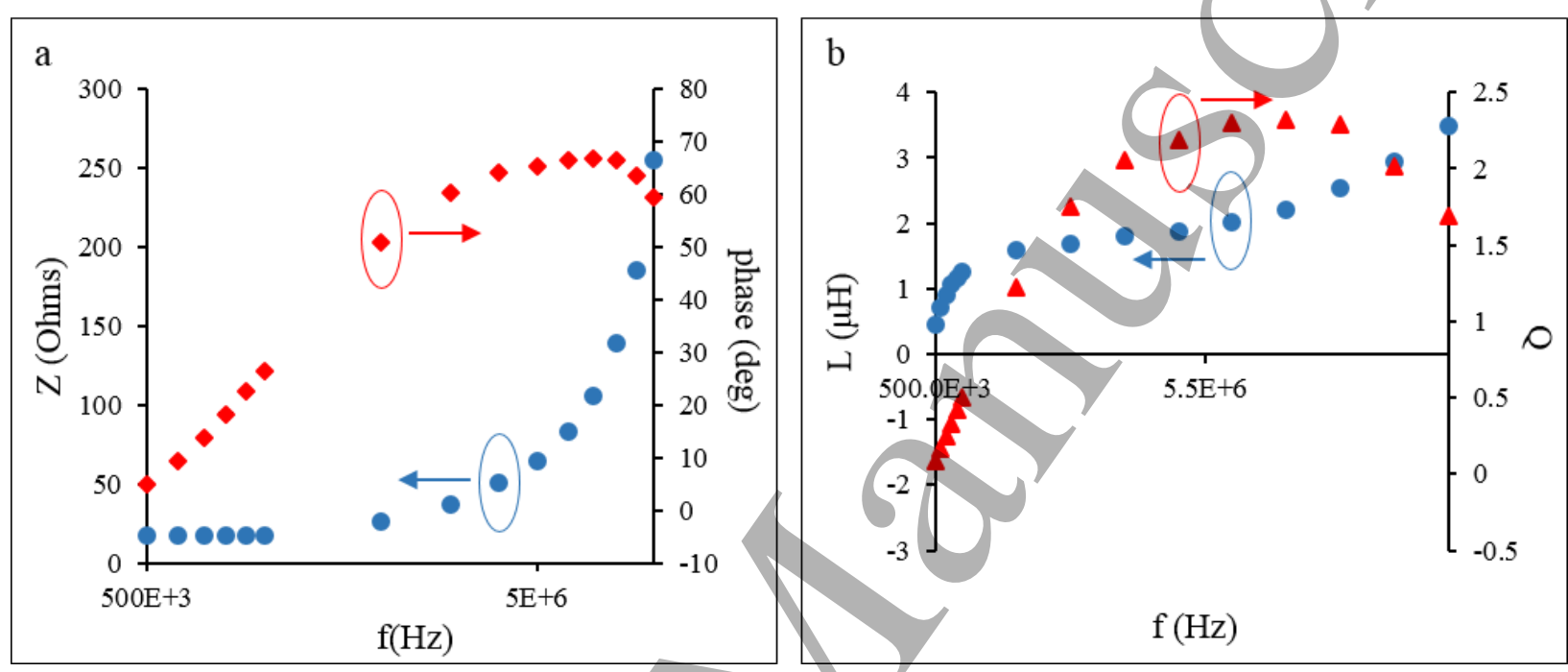

Figure 5. (a) Measured magnitude and phase of the complex impedance vector. (b) Inductance and quality factor $\mathrm{Q}$ of the system. For both cases, a logarithic scale is used for the horizontal axis of frequency.

\subsection{NEURON modelling}

As described in the methods section, an axon $70 \mu \mathrm{m}$ in length and $10 \mu \mathrm{m}$ in diameter is placed at a plane $10 \mu \mathrm{m}$ away from the centre of the microcoil modelled with NEURON software. The code was alternated to describe our design, with the measured R,L,C values and the specific spatial distribution of electric field, which is determined by the geometry of the micro-coils under study. The planar distribution of the electric fiels is presented in figure 6(a), while in figure 6(b) the spatial distribution of the $x$ component of the electric field is illustrated, both of them for the plane where $\mathrm{z}=10 \mu \mathrm{m}$.

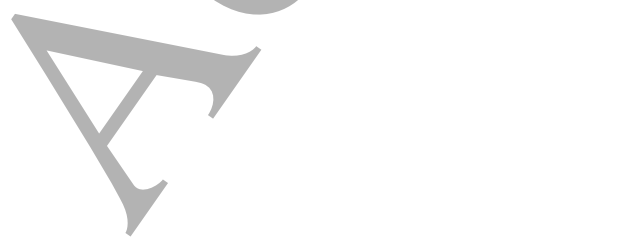



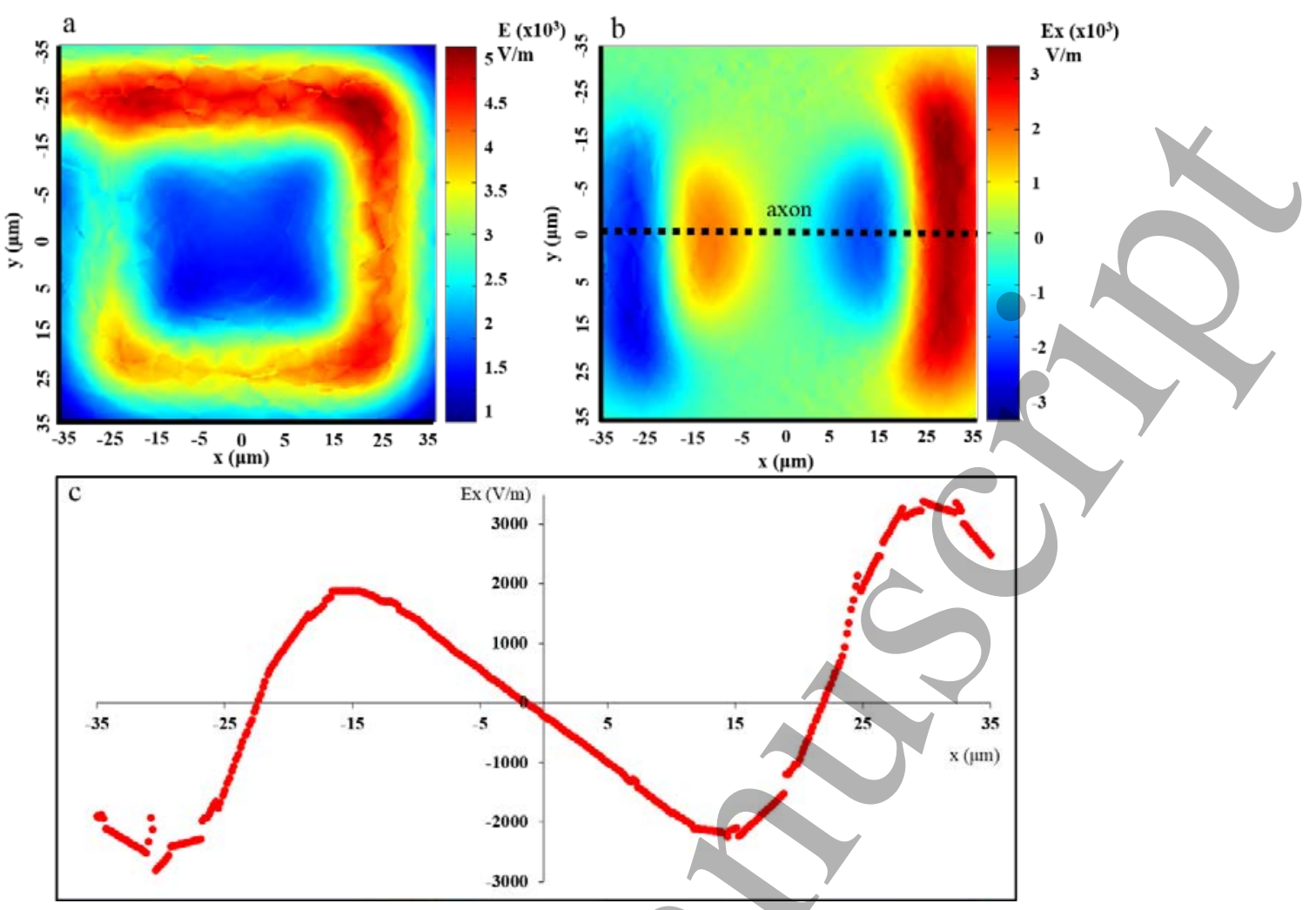

Figure 6. Planar distribution of (a) electric field (E) and (b) x component of electric field (Ex), for a distance of $10 \mu \mathrm{m}$ above the micro-coils. (c) Plot of $\mathrm{x}$ component of electric field along $\mathrm{x}$, for a specific value of $\mathrm{y}$.

The graph shows also the relative position of the axon to the micro-coil. Finally, figure 6(c) is giving the plot of Ex along the increase of $\mathrm{x}$, for $\mathrm{y}=0$ where the axon is located. The values of this plot are exported from COMSOL and used in our NEURON code. The transmembrane potential elicited in the axon modelled, is presented in figure 7 along with time, revealing the activation of the cell for a stimulus of $200 \mathrm{mV}$ with a duration of $0.4 \mathrm{~ms}$.

Figure 7. Stimulus manages to activate the neural cell. The activation of the cell is illustrated by the transmambrane potential elicited in the axon over time. 
${ }^{43} 360$

${ }_{45} 261$

46262

47263

48264

${ }^{49} 265$

51266

\subsection{Testing with a phantom gel}

At first, we performed an electric characterisation of the gel that is presented in figure 8 in otder to define the its electric permitivity and resistivity. The electric characterization of the gel, was performed with the parallel plate capacitor method, appropriate for the frequency range of $500 \mathrm{kHz}-1 \mathrm{MHz}$.

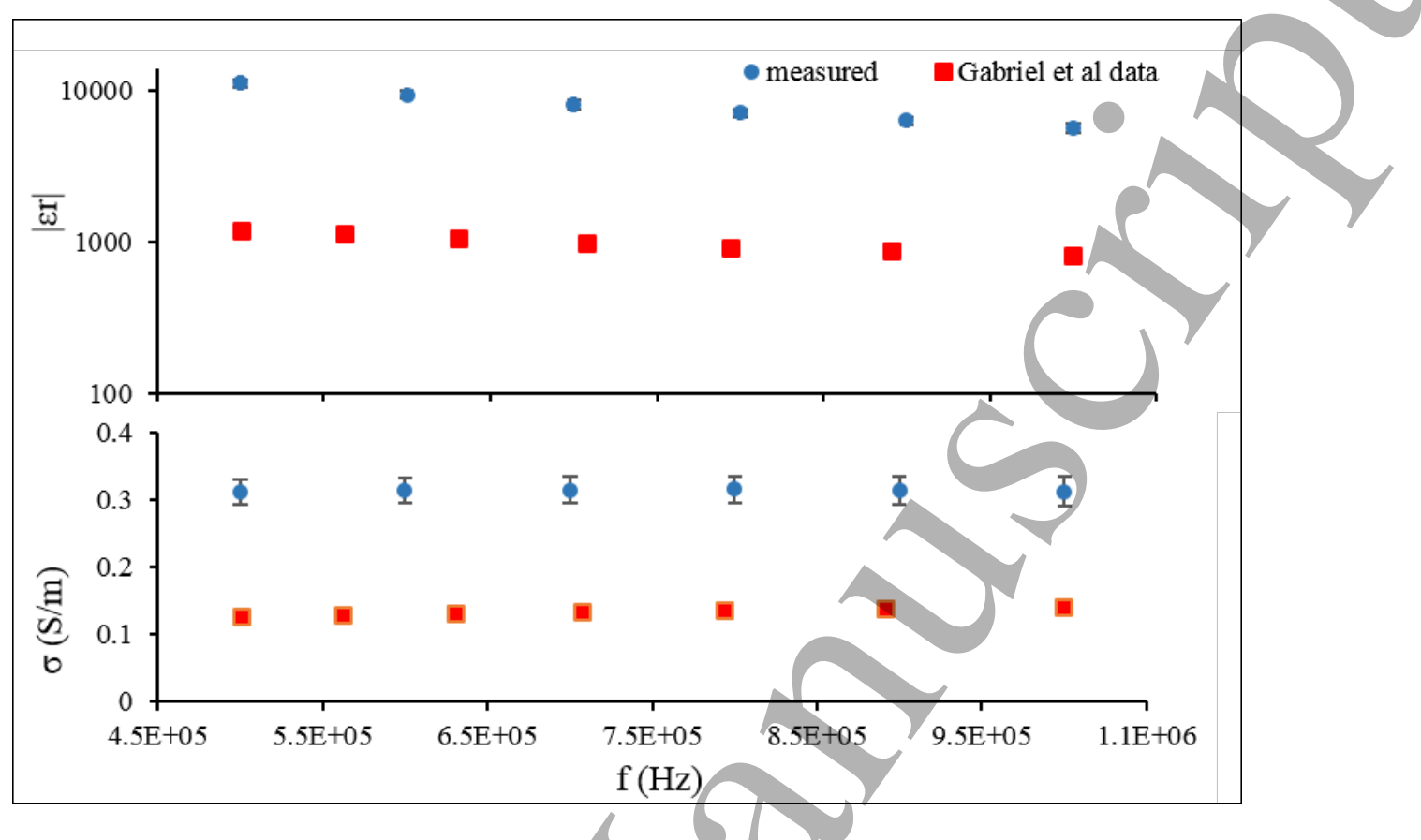

Figure 8. Electric permittivity and conductivity values for the frequency range of $500 \mathrm{kHz}-1 \mathrm{MHz}$. Measured values of the fabricated phantom compared with measured values in real neural tissue. Error bars representing standard error.

The gel is set as a dielectric between the metallic plates of a capacitor with a thickness of $3 \mathrm{~mm}$, so as to have negligible impact on the measurements from the air trapped between the gel and the metallic plates. Three sets of measurements of electric permittivity $\varepsilon_{\mathrm{r}}$ and electric conductivity $\sigma$ were taken and their mean values, displayed with circle markers in the graphs, are directly compared to the values of real biological brain tissue, given by [25]. The relevant biologically expected values, displayed with square markers, are the mean values of white and grey matter for every frequency.

At the next step, a chip with a single micro-coil geometry, covered with Parylene-C, was used in order to identify the induced current density evoked into the gel by the activation of the micro-coil. The signal used for the biasing of the micro-coil was an AC signal of $500 \mathrm{kHz}$, for a number of different amplitudes between 20$200 \mathrm{mV}$. A burst of rectangular pulses was also tested as a biasing signal, but was not preferred for this experiment, due to charging phenomena on the gel. The measurements were performed with the use of Keithley semiconductor parameter analyser and the use of a probe station. The probe was also set to a number of different positions above the centre of the microcoil, starting from the surface of the Parylene- $C$ film $(z=0)$ to a distance of $1000 \mu \mathrm{m}$, set with the use of a micropositioner and a microscope. The results are displayed in figure 9 and are part of one set of measurements inside the gel. For every position of $\mathrm{z}$, four different measurements were taken for the different voltages applied (peak to peak values). Repetitions of the measurements have been performed, but decided not to be included, as there was a difficulty in relocating the needle into the $x, y . z$ position due to the semi-solid consistency of the gel and could be misleading. Data from the repetition of the experiments, for a different $\mathrm{x}, \mathrm{y}, \mathrm{z}$ position in the gel and above the micro-coil, as well as for different biasing 
frequencies, which are not included in this study, show similar behaviour and induced currents in the range of $10 \mu \mathrm{A}$.

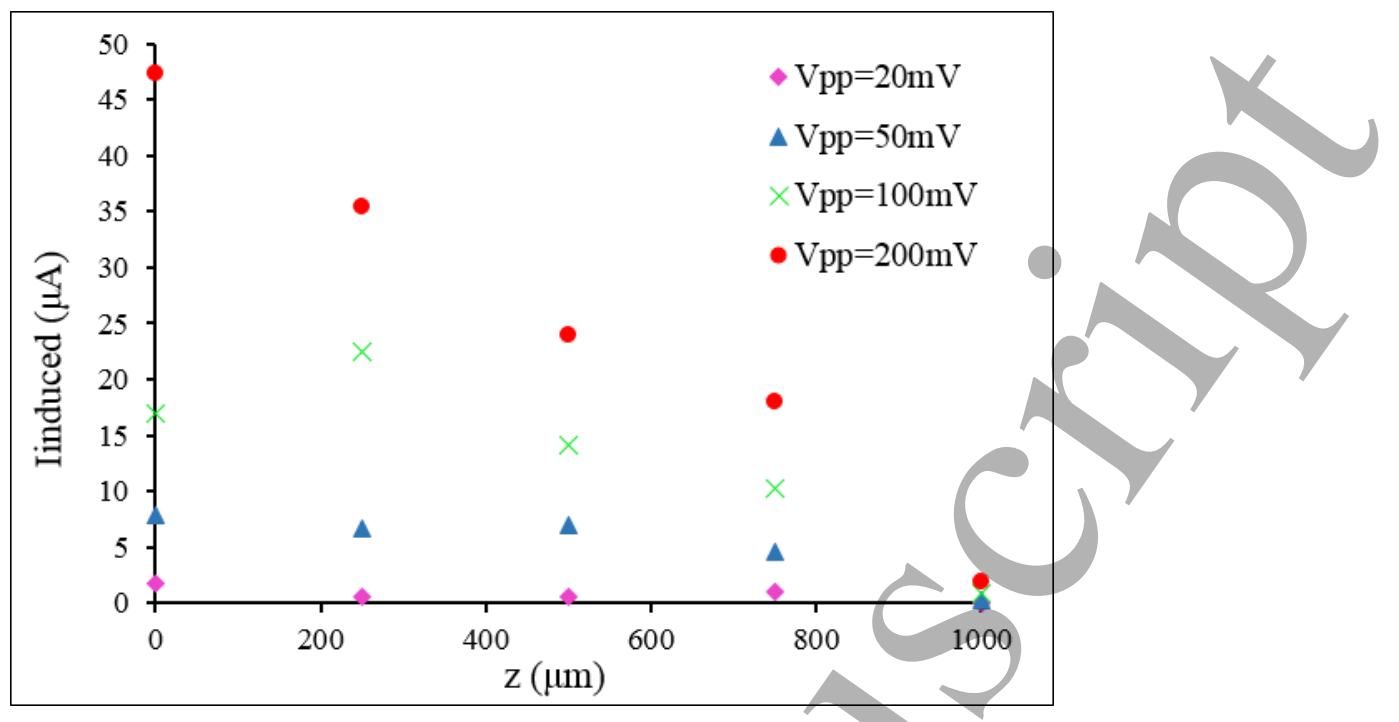

Figure 9. The current induced in the phantom solution, for a different nuber of positions away from the microcoil and for a different setting of peak to peak applied voltage used as bias to the micro-coils. All the measurements were performed for the frequency of $500 \mathrm{kHz}$.

\section{Discussion}

The impedance of the prototype device defined experimentally, in combination with the results of the spatial distribution of the electric and magnetic field resulting from the electromagnetic study in COMSOL, are providing us with useful information regarding the characteristic behaviour of the system. These characteristics are then inserted into NEURON environment, in order to simulate the magnetic stimulation mechanism. In reality, cells or biological tissue reacts on the electric field, rather than on the magnetic field itself. This is why the induced electric field values are needed for the simulation in NEURON. The simulation shows that a simplified cell geometry, placed in a distance similar to the realistic case, feels the presense of the induced electric field and an action potential is elicited and propagates along the axon.

Following the simulation study, a proof of concept of our device was experimentally tested with a phantom gel. The electric characterisation of the phantom gel, reveals that the electric conductivity values for a range of frequencies are very close to that of brain tissue, while the electric permittivity results could not reach the same extend of similarity. However, the value of conductivity is influencing more significantly the induced current density in a leaky dielectric. Specifically, the current density in a leaky dielectric is given by equation (3).

$$
\mathbf{J}=\mathbf{J}_{\text {free }}+\mathbf{J}_{\text {bound }}=\sigma \mathbf{E}+\frac{\partial \mathbf{P}}{\partial \mathrm{t}}+\nabla \times \mathbf{M}
$$

$\mathbf{J}$ is the current density which consist of two parts: $\mathbf{J}_{\text {free }}$ current density by free carriers and the $\mathbf{J}_{\text {bound }}$ current density attributed on the relocation of bound charges. $\mathbf{E}$ the applied electric field vector, $\mathbf{P}$ the polarisation field vector produced by the dielectric and $\mathbf{M}$ the magnetization vector of the material. Since the material has not magnetic properties, the last part of equation (3) could be omitted. For the frequency range of interest, and by moving to the frequency domain as we study harmonic signals, a simple comparison between the two terms is enough to give the dominant factor in the current density of the dielectric: 


$$
\mathbf{J}_{\text {free }}>>\mathbf{J}_{\text {bound }}
$$

The explanation arises by equations (5) and (6) below. $\mathrm{J}_{\text {bound }}$ in equation (6) takes a range of values, defined by the frequency $\omega$ of the harmonic signal, but in any case gives a $\mathbf{J}_{\text {bound }}$ smaller at least by an order of magnitude from $\mathbf{J}_{\text {free. }}$

$$
\begin{gathered}
\mathbf{J}_{\text {free }}=\sigma \mathbf{E} \approx\left(10^{-1} \frac{\mathrm{S}}{\mathrm{m}}\right) \mathbf{E} \approx 10^{-1} \mathbf{E} \\
\mathbf{J}_{\text {bound }}=i \omega \mathbf{P}=i \omega \varepsilon_{o}\left(\varepsilon_{r}-1\right) \mathbf{E} \approx\left(10^{5} s^{-1}\right)\left(10^{-12} \frac{\mathrm{F}}{\mathrm{m}}\right) 10^{4} \mathbf{E} \approx 10^{-3} \mathbf{E}
\end{gathered}
$$

As a result, despite the electric permitivity significant difference between measured and biological values, it is possible to use the suggested phantom and extract safe results. Regarding the difference in conductivity, which should be also taken into consideration, it is easy to assign more realistic values to our results by a simple multiplication, as the current depends linearly on the value of conductivity.

The measurment of the induced current in the gel, in figure 9 proves the functionality of the device and as expected shows a decreasing tendency as we move away from the micro-coil surface. The amplitude of the induced current is extracted in the steady state with the use of a harmonic signal of $500 \mathrm{kHz}$. The maximum value is received, for $\mathrm{z}=0$ just above the Parylene-C surface, while for every position $\mathrm{z}$, the induced current increases along with the increase of the biasing voltage amplitude. A direct comparison of these results with those of study[26], show that the proposed platform produces equivalent current amplitudes to electrical pulses applied extracellularly in the cell medium. More specifically, that study reported that neural cells of the spinal border cells or motoneurons, have their lowest threshold of activation (for a minimum distance from the cell) at stimulus amplitudes of $0.15-0.20 \mu \mathrm{A}$ and $0.35-0.40 \mu \mathrm{A}$ respectively. Figure 9 shows that for the minimum distance the results are significantly higher, even by interpolation to the more realistic expected values of conductivity (devide by a factor of 3 the conductivity values received from figure 8).

However, the threshold of cell activation is not determined only by the amplitude of the stimulus but also by its duration and is given by the strength-duration relation. The strength-duration curve is not fully understood for the magnetic stimulation mechanism[17]. Therefore, our approach in the real bio-experiment would be to start from a specific stimulus duration and run over a range of stimulus amplitudes, which should be below the deterioration threshold of the micro-coils. This procedure will be repeated for gradually alternating the stimulus duration, until cell activation will be observed. Furthermore, in a realistic scenario of cell stimulation, a pulsed method of stimulation could be combined with a sinusoidal signal of higher frequency, as in [19], where a burst of sinusoidal periods led to suppression of neuronal activity.

In the study presented here, stimulus duration has been tested with NEURON and cell stimulation for purely pulsed stimuli was observed with amplitudes up to $200 \mathrm{mV}$ and durations between $2 \mu \mathrm{s}-2 \mathrm{~ms}$. Stimulus with an amplitude of $200 \mathrm{mV}$ and duration of $1 \mu \mathrm{s}$ did not elicit cell activity. The range of stimuli we tested was based on similar studies of microscale magnetic stimulation[17-19].

Finally, micro-coils should be able to work over a wide range of biological preparation, a fact that implies that the bioexperiment itself would determine the specific needs for the characteristics of the system. In case further improvement of the results is needed to achieve cell stimulation, this could be achieved by thicker coils, with larger cross section, able to hold a higher current capacity and produce stronger electromagnetic field. Also, a signal with a steeper time variance, such as a pulse of same amplitude and frequency, would result in an even higher induced current magnitude as describes by Faraday's law. 


\section{Conclusions}

This study described the development of a bio-device consisting of 36 separately addressable micro-inductors, designed to create magnetic profiles with microscale resolution. We demonstrate the fabrication from the chip level to a biocompatible package. The performance of the device is tested both numerically and experimentally. In summary, the RLC characteristics of the system, received from the electric characterization, are used to calculate the temporal component of the stimulating effect on NEURON. The induced electric field, extracted from COMSOL calculations is also imported to NEURON to define the spatial component of the stimulation. The numerical study showed that an action potential is created and propagated along the axon of a neural cell, placed at $10 \mu \mathrm{m}$ away from the inductor. Furthermore, a phantom gel with similar electric properties as the brain tissue was developed and used to quantify the current induced by the activation of a micro-coil. This work sets up the scheme for alternative methods to control the behaviour of neural cells in vitro, having at the same time as a main advantage the non-invasiveness of the method. Future steps of this work concern a successful biological validation of the proposed platform, as well as a detailed study on the electrothermal phenomena (Joule heating, electromigration) which lead to the degradation of the micro-coils and limit their durability. At the same time, the morphology of the chip could be easily transferred to flexible substrates, finding possible applications in the field of implantable bio-devices.

\section{Acknowledgments}

We would like to acknowledge the financial support of the A.G. Leventis Foundation and EPSRC EP/L020920/1.

\section{References}

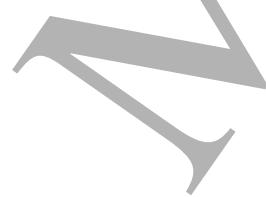

[1] Frazier A B, O’Brien D P and Allen M G 1993 Two dimensional metallic microelectrode arrays for extracellular stimulation and recording of neurons [1993] Proc. IEEE Micro Electro Mech. Syst. 195200

[2] Fernández E, Greger B, House P a, Aranda I, Botella C, Albisua J, Soto-Sánchez C, Alfaro A and Normann R a 2014 Acute human brain responses to intracortical microelectrode arrays: challenges and future prospects. Front. Neuroeng. 724

[3] Zeitler R, Fromherz P and Zeck G 2011 Extracellular voltage noise probes the interface between retina and silicon chip Appl. Phys. Lett. 99

[4] Zemelman B V., Lee G A, Ng M and Miesenböck G 2002 Selective photostimulation of genetically chARGed neurons Neuron 33 15-22

[5] Häusser M 2014 Optogenetics: the age of light Nat. Methods 11 1012-4

[6] Huang H, Delikanli S, Zeng H, Ferkey D M and Pralle A 2010 Remote control of ion channels and neurons through magnetic-field heating of nanoparticles Nat. Nanotechnol. 5 602-6

[7] Shapiro M G, Homma K, Villarreal S, Richter C-P and Bezanilla F 2012 Infrared light excites cells by changing their electrical capacitance Nat. Commun. 3736

[8] Barker A T, Jalinous R and Freeston I L 1985 Non-Invasive Magnetic Stimulation of Human Motor Cortex Lancet 325 1106-7 
[9] McNamara B, Ray J L, Arthurs O J and Boniface S 2001 Transcranial magnetic stimulation for depression and other psychiatric disorders Psychol. Med. 31 1141-6

[10] George M S, Nahas Z, Lisanby S H, Schlaepfer T, Kozel F A and Greenberg B D 2003 Transcranial magnetic stimulation. Neurosurg. Clin. N. Am. 14 283-301

[11] Mäkelä J 2015 Navigated transcranial magnetic stimulation in clinical practice and research Navigated transcranial magnetic stimulation in clinical practice and research Int. J. Bioelectromagn. 17 7-12

[12] Accoto D, Francomano M T, Rainer a, Trombetta M, Rossini P M and Guglielmelli E 2013 An implantable neural interface with electromagnetic stimulation capabilities. Med. Hypotheses $\mathbf{8 1} 322-7$

[13] Tischler H, Wolfus S, Friedman A, Perel E, Pashut T, Lavidor M, Korngreen A, Yeshurun Y and BarGad I 2011 Mini-coil for magnetic stimulation in the behaving primate J. Neurosci. Methods $194242-$ 51

[14] Pashut T, Wolfus S, Friedman A, Lavidor M, Bar-Gad I, Yeshurun Y and Korngreen A 2011 Mechanisms of magnetic stimulation of central nervous system neurons. PLoS Comput. Biol. 7 e1002022

[15] Basham E, Yang Z and Liu W 2008 Circuit and Coil Design for In Vitro Magnetic Neural Stimulation Systems

[16] Constandinou T G, Georgiou P, Prodromakis T and Toumazou C 2010 A CMOS-based lab-on-chip array for the combined magnetic stimulation and opto-chemical sensing of neural tissue 12th International Workshop on Cellular Nanoscale Networks and their Applications (CNNA 2010) (IEEE) pp 1-6

[17] Bonmassar G, Lee S W, Freeman D K, Miloslav P, Fried S I and Gale J T 2012 Microscopic magnetic stimulation of neural tissue Nat. Commun. 3921

[18] Park H J, Bonmassar G, Kaltenbach J A, Machado A G, Manzoor N F and Gale J T 2013 Activation of the central nervous system induced by micro-magnetic stimulation. Nat. Commun. 42463

[19] Lee S W and Fried S I 2015 Suppression of Subthalamic Nucleus Activity by Micromagnetic Stimulation IEEE Trans. Neural Syst. Rehabil. Eng. 23 116-27

[20] Lee S W, Fallegger F, Casse B D F and Fried S I 2016 Implantable microcoils for intracortical magnetic stimulation Sci. Adv. 2 e1600889-e1600889

[21] Carnevale N T and Hines M L 2004 Preface to The NEURON Book Neuron 1-762

[22] Hartsgrove G, Kraszewski A and Surowiec A 1987 Simulated biological materials for electromagnetic radiation absorption studies. Bioelectromagnetics 8 29-36

[23] Moulin J, Woytasik M, Grandchamp J-P, Dufour-Gergam E and Bosseboeuf A 2006 High current densities in copper microcoils: influence of substrate on failure mode Current (Stresa, Italy) pp 26-8

[24] Maccabee P J, Amassian V E, Eberle L P and Cracco R Q 1993 Magnetic coil stimulation of straight and bent amphibian and mammalian peripheral nerve in vitro: locus of excitation. J. Physiol. 460 20119

[25] Gabriel S, Lau R W/and Gabriel C 1996 The dielectric properties of biological tissues: II. Measurements in the frequency range $10 \mathrm{~Hz}$ to $20 \mathrm{GHz}$. Phys. Med. Biol. 41 2251-69

[26] Gustafsson B and Jankowska E 1976 Direct and indirect activation of nerve cells by electrical pulses applied extracellularly. J. Physiol. 258 33-61 